\title{
Time-resolved structural biology benefits from complementary methods
}

Allen Milster Orville ${ }^{1}$, Franklin Fuller ${ }^{2}$, Sheraz Gul ${ }^{2}$, Jan Kern² ${ }^{2}$ Aaron Brewster ${ }^{2}$, Nicholas Sauter ${ }^{2}$, Uwe Bergmann ${ }^{3}$, Roberto AlonsoMori $^{4}$, Vittal Yachandra ${ }^{2}$, Junko Yano ${ }^{2}$

${ }^{1}$ Life Sciences, Diamond Light Source, Didcot, United Kingdom, ${ }^{2}$ Molecular Biophysics and Integrated Bioimaging Division, Lawrence Berkeley National Laboratory, Berkeley, United States, ${ }^{3}$ Stanford PULSE Institute, SLAC National Accelerator Laboratory, Menlo Park, United States, ${ }^{4}$ LCLS, SLAC National Accelerator Laboratory, Menlo Park, United States E-mail: allen.orville@diamond.ac.uk

More than 110,500 atomic models based on X-ray crystallography are available in the protein data bank (PDB) and nearly all of them are from diffraction data collected from samples held at $100 \mathrm{~K}$. These cryogenic models provide critical and important functional insights. But, they are snapshots of ground-state molecules or from trapped complexes; whereas biology is dynamic. Indeed, life is governed by the complex interplay within and between macromolecules (proteins, nucleic acids, lipids and carbohydrates) that yield function. Thus, an outstanding frontier challenge in structural biology is to determine time-resolved crystal structures directly from systems engaged in catalysis.

To fully realize time-resolved/dynamic structural biology, one must understand as much function as possible from the same sample and/or molecular state. For example, AMO developed (polarized) single-crystal electronic absorption and resonance Raman spectroscopies that were correlated with synchrotron-based X-ray crystallography. These methods provide complementary data for electronic and atomic structure that can be obtained from the same volume of crystalline sample. More recently, we have developed on-demand acoustic sample delivery strategies to more efficiently collect fully-correlated serial femtosecond crystallography (SFX) and X-ray emission spectroscopic (XES) data from the same XFEL pulse.

Acoustic droplet ejection (ADE) is a general, touchless, on-demand method that uses focused sound waves to eject picoliter to nanoliter volume droplets from the surface of one liquid to another location. Our injector delivers discrete sample amounts onto a moving conveyor belt. It is optimized for photochemical and chemical reactions over a wide range of time scales and enables X-ray diffraction in the forward direction simultaneously with XES measurements at 90 degrees. Studies with photosystem II, the phytochrome photoreceptor, and ribonucleotide reductase R2 illustrate the power and versatility of this method.

[1] Roessler, C. G. et al (2016) Structure 24, 631-640.

[2] Fuller, F. D. and Gul S. et al (2017) Nature Methods (in press).

[3] Young, I. D., Ibrahim, M., and Chatterjee, R. et al (2016) Nature 540, 453-457.

Keywords: serial femtosecond crystallography, $\mathrm{X}$-ray emission spectroscopy, time-resolved structural biology 